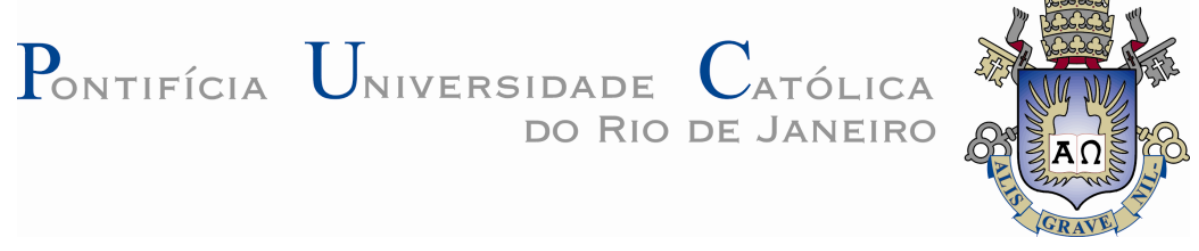

Roberto Carlos Chucuya Huallpachoque

\title{
Análise Numérica do Escoamento Bifásico Horizontal em \\ Padrão de Golfadas Estatisticamente Permanente
}

Dissertação de Mestrado

Dissertação apresentada como requisito parcial para obtenção do grau de Mestre pelo Programa de Pós-graduação em Engenharia Mecânica do Departamento de Engenharia Mecânica da PUCRio.

Orientadora: Profa Angela Ourivio Nieckele

Rio de Janeiro

Setembro 2010 


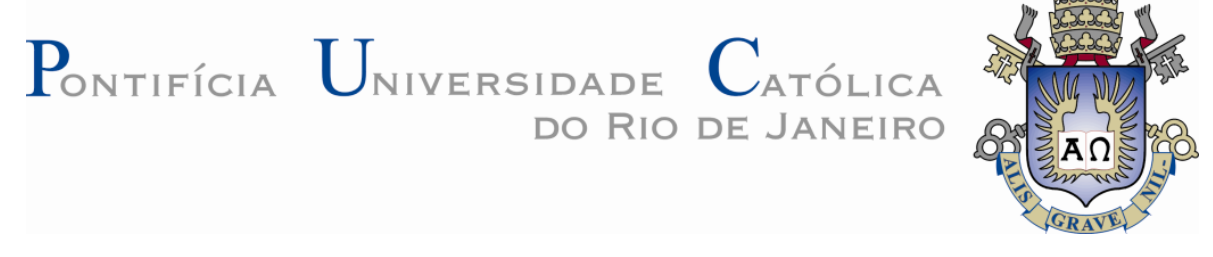

Roberto Carlos Chucuya Huallpachoque

\title{
Análise Numérica do Escoamento Bifásico Horizontal em Padrão de Golfadas Estatisticamente Permanente
}

\begin{abstract}
Dissertação apresentada como requisito parcial para obtenção do título de Mestre pelo Programa de PósGraduação em Engenharia Mecânica do Centro Técnico Científico da PUC-Rio. Aprovada pela Comissão Examinadora abaixo assinada.
\end{abstract}

Profa. Angela Ourivio Nieckele

Orientadora

Departamento de Engenharia Mecânica - PUC-Rio

Prof. José Alberto dos Reis Parise Departamento de Engenharia Mecânica - PUC-Rio

Prof. Antônio Carlos Bannwart

Universidade Estadual de Campinas

Prof. José Eugenio Leal

Coordenador Setorial do Centro Técnico Científico - PUC-Rio

Rio de Janeiro, 16 de setembro de 2010 
Todos os direitos reservados. É proibida a reprodução total ou parcial do trabalho sem autorização da universidade, do autor e do orientador.

\section{Roberto Carlos Chucuya Huallpachoque}

Graduou-se em Engenharia em Energia na Universidad Nacional del Santa (Chimbote - Ancash - Perú) no ano de 2006. Atualmente trabalhando na simulação de escoamento multifasico.

Ficha Catalográfica

Chucuya Huallpachoque, Roberto Carlos

Análise numérica do escoamento bifásico horizontal em padrão de golfadas estatisticamente permanente / Roberto Carlos Chucuya Huallpachoque ; orientadora: Angela Ourivio Nieckele. - 2010.

116 f. : il. (color.) ; $30 \mathrm{~cm}$

Dissertação (mestrado)-Pontifícia Universidade Católica do Rio de Janeiro, Departamento de Engenharia Mecânica, 2010.

Inclui bibliografia

1. Engenharia mecânica - Teses. 2. Padrão golfadas. 3. Tubulação horizontal. 4. Analise estatística. I. Nieckele , Angela Ourivio. II. Pontifícia Universidade Católica do Rio de Janeiro. Departamento de Engenharia Mecânica. III. Título. 
"Si un hombre es perseverante, aunque sea duro de entendimiento, se hará inteligente; y aunque sea débil se transformará en fuerte" 


\title{
Agradecimentos
}

\author{
À Profa. Angela Ourivio Nieckele, pela paciência, dedicação e por sempre \\ ter me exigido o melhor de mim.
}

Às agências CAPES, CNPq e à PUC-Rio pelos auxílios concedidos, sem os quais este trabalho não poderia ter sido realizado.

À minha família, Mãe e meus Irmãos: Gladys, Raquel, Claudia e Jorge pelo apoio incondicional e ânimos no transcurso destes dois anos, vocês são a referencia mais importante em minha vida.

À Sylvia que sempre esteve comigo todos os dias, com seu sorriso e brilho especial que a faz ser a pessoa cativante que me conquistou. Sua alegria e amor me fazem uma pessoa melhor a cada dia.

Aos meus amigos da favelinha andar 6 , em especial a José F. e José M. 


\section{Resumo}

Chucuya Huallpachoque, Roberto Carlos; Nieckele, Angela Ourivio. Análise Numérica do Escoamento Bifásico Horizontal em Padrão de Golfadas Estatisticamente Permanente. Rio de Janeiro, 2010. 116p. Dissertação de Mestrado - Departamento de Engenharia Mecânica, Pontifícia Universidade Católica do Rio de Janeiro.

O escoamento bifásico no regime de golfadas é um padrão de escoamento que requer esforço em sua caracterização e modelagem, devido às características marcantes da distribuição espacial das fases, que gera intermitência ao escoamento. Este escoamento ocorre na presença de uma grande faixa de vazões de gás e líquido, em tubulações de diâmetro médio e pequeno, com variação periódica da densidade, fração de vazio e pressões na seção transversal da tubulação. No presente trabalho estuda-se numericamente o regime de golfadas ao longo de tubulações horizontais utilizando-se o Modelo de Dois Fluidos. Uma análise detalhada das estatísticas do escoamento é realizada com a caracterização dos principais parâmetros de uma golfada, como comprimento, velocidade da frente e cauda e frequiência de passagem das golfadas ao longo da tubulação. Os dados obtidos das simulações são analisados através de histogramas de distribuição assim como de funções densidade de probabilidade (PDF) das variáveis hidrodinâmicas, destacando-se o caráter estocástico do escoamento no padrão de golfadas. Adicionalmente, comparações com dados de trabalhos experimentais da literatura foram realizadas com concordância muito boa.

\section{Palavras-chave}

Padrão Golfadas; Tubulação Horizontal; Analise Estatística. 


\section{Abstract}

Chucuya Huallpachoque, Roberto Carlos; Nieckele, Angela Ourivio (Advisor). Numerical Analysis of Flow Pattern in Horizontal Twophase slug flow Statistically Permanent, Rio de Janeiro, 2010. 116p. MSc. Dissertation - Departamento de Engenharia Mecânica, Pontifícia Universidade Católica do Rio de Janeiro.

Slug flow is a two-phase flow pattern that requires large effort in its characterization and modeling, due to special characteristics of the phase's spatial distribution, which causes flow intermittency. This flow occurs in a wide range of gas and liquid flow rates in pipes of medium and small diameters, with periodic variation of density, void fraction and pressures in pipe-cross-section. This work presents a numerical study of the slug regime through horizontal pipes using the Two-Fluid Model. A detailed statistical analysis of the flow was carried out with characterization of main slug parameters, such as slug length, front and tail velocities and slug frequency along the pipeline. The numerical results were analyzed through distribution histograms as well as probability density function (PDF) of the hydrodynamic variables, showing the stochastic characteristic of slug flow pattern. Further, comparisons with experimental data from the literature were performed, showing very good agreement.

\section{Keywords}

Slug Flow; Horizontal Pipeline; Statistical Analysis 


\section{Sumário}

$\begin{array}{lr}\text { 1. Introdução } & 18\end{array}$

1.1. Regime de Golfadas $\quad 21$

1.2. Objetivo 23

1.3. Organização do Trabalho 24

2. Revisão Bibliográfica 25

2.1. Mapas de Padrão de Escoamento 25

2.2. Estudos Experimentais 28

2.3. Estudos Numéricos 32

3. Modelagem Matemática 35

3.1. Modelo de Dois Fluidos $\quad 35$

3.2. Condições de Contorno e Iniciais 41

3.2.1. Mapa de Padrão e Condição para Sistema Bem Posto 42

3.3. Variáveis do Escoamento Estatisticamente Permanente 45

3.3.1. Função Densidade de Probabilidade: PDF 45

4. Método Numérico 50

4.1. Fração Volumétrica 51

4.2. Velocidades 52

4.3. Pressão 54

4.4. Condições de Contorno 56

4.5. Malha Computacional e Passo de Tempo 57

4.6. Critério de Convergência 58

4.7. Procedimento de Solução 59 
4.7.1 Procedimento Seqüencial $\quad 59$

4.7.2 Procedimento em Bloco 60

$\begin{array}{ll}\text { 4.7.3. Algoritmo TDMA } & 60\end{array}$

4.7.4. Algoritmo TDMA em Blocos 61

4.8. Formação da Golfada 62

4.9. Cálculo dos Parâmetros Médios das Golfadas 63

5. Resultados 66

$\begin{array}{ll}\text { 5.1. Configuração } 1 & 67\end{array}$

5.1.1. Comprimento das Golfadas 70

5.1.2. Velocidade de Translação das Golfadas 80

5.1.3. Freqüência das Golfadas 83

5.1.4. Distribuição de Pressão 85

5.1.5. Distribuição de Velocidades 87

5.2. Configuração $2 \quad 88$

5.2.1. Velocidade de Translação das Golfadas 92

5.2.2. Comprimento da Golfada Líquida e da Bolha de Taylor 95

5.2.3. Freqüência das Golfadas 100

5.2.4. Distribuição de Pressão 103

6. Comentários Finais 105

$\begin{array}{ll}\text { Referências Bibliográficas } & 108\end{array}$

$\begin{array}{ll}\text { Apêndice A } & 112\end{array}$ 


\section{Lista de tabelas}

Tabela 2.1 - Comprimento da Golfada Líquida 31

Tabela 3.1 - Fórmulas para o cálculo do fator de atrito 39

Tabela 5.1 - Propriedades dos Fluidos $\quad 66$

Tabela 5.2 - Condições Experimentais de Operação 67

Tabela 5.3 - Comprimentos Médios das Golfadas em Cada Coordenada 74

Tabela 5.4 - Determinação do Erro Padrão. Configuração 1

Tabela 5.5 - Valores dos Parâmetros $C_{0}$ e $U_{d}$

Tabela 5.6 - Correlaçoes de Lockhart-Martinelli 86

Tabela 5.7 - Parâmetros de Lockhart-Martinelli. 87

Tabela 5.8 - Queda de Pressão Média. Configuração 1

Tabela 5.9 - Condições Experimentais de Operação 89

Tabela 5.10 - Velocidade Média da Frente e Cauda da Golfada Líquida 92

Tabela 5.11 - Relação entre a Velocidade Média da Cauda e Frente da Golfada 93

Tabela 5.12 - Comprimento da Golfada líquida e Bolha de Taylor 95

Tabela 5.13- Determinação do Erro Padrão. Configuração 299

Tabela 5.14 - Queda de Pressão Média. Configuração 2 


\section{Lista de figuras}

Figura 1.1 - Padrões de escoamento para os fluxos horizontal e vertical.

Figura 1.2 - Sistema de produção de óleo na indústria do Petróleo. 20

Figura 1.3 - Unidade básica de uma golfada.

Figura 1.4 - Escoamento em golfada em tubulação horizontal e vertical.

Figura 1.5 - Formação de golfada em tubulação inclinada.

23

Figura 2.1 - Mapa dos padrões de escoamento, Mandhane (1974)

26

Figura 2.2 - Mapa dos padrões de escoamento, Taitel e Dukler (1976)

Figura 3.1 - Esquema da tubulação e seção transversal.

Figura 3.2 - Condições de contorno utilizadas.

Figura 3.3 - A Função Densidade de Probabilidade Normal.

Figura 3.4 - A Função Densidade de Probabilidade Log-Normal.

Figura 4.1 - Volumes de controle. (a) fração volumétrica e pressão (b) velocidades.

Figura 4.2 - Volume de controle da fronteira do domínio: (a) na entrada (b) saída.

Figura 4.3 - Ilustração da medição da velocidade cada golfada. (a) frente (b) calda.

Figura 5.1 - Mapa de Padrões de Escoamento da Configuração 1.

Figura 5.2 - Evolução do Hold-up Líquido em Espaço e Tempo.

Caso 1: $U_{s L}=0,41 \mathrm{~m} / \mathrm{s}$ e $U_{s G}=2,36 \mathrm{~m} / \mathrm{s}$.

Figura 5.3 - Evolução do Hold-up Líquido em Espaço e Tempo.

Caso 2: $U_{s L}=0,61 \mathrm{~m} / \mathrm{s}$ e $U_{s G}=2,55 \mathrm{~m} / \mathrm{s}$.

Figura 5.4 - Evolução do Hold-up Líquido em Espaço e Tempo.

Caso 3: $U_{s L}=0,61 \mathrm{~m} / \mathrm{s}$ e $U_{s G}=4,64 \mathrm{~m} / \mathrm{s}$.

Figura 5.5 - Histograma do Comprimento das Golfadas ao Longo da 
Tubulação. Caso 1: $U_{s L}=0,41 \mathrm{~m} / \mathrm{s}$ e $U_{s G}=2,36 \mathrm{~m} / \mathrm{s}$.

Figura 5.6 - Histograma do Comprimento das Golfadas ao Longo da Tubulação. Caso 2: $U_{s L}=0,61 \mathrm{~m} / \mathrm{s}$ e $U_{s G}=2,55 \mathrm{~m} / \mathrm{s}$.

Figura 5.7 - Histograma do Comprimento das Golfadas ao Longo da Tubulação. Caso 3: $U_{s L}=0,61 \mathrm{~m} / \mathrm{s}$ e $U_{s G}=4,64 \mathrm{~m} / \mathrm{s}$.

Figura 5.8 - Distribuição do Comprimento Medio das Golfadas ao Longo da Tubulação. Casos 1, 2 e 3

Figura 5.9 - Evolução do Comprimento da Golfada Ajustado por Funções de Densidade de Probabilidade (PDF).

Caso 1: $U_{s L}=0,41 \mathrm{~m} / \mathrm{s}$ e $U_{s G}=2,36 \mathrm{~m} / \mathrm{s}$.

Figura 5.10 - Evolução do Comprimento da Golfada Ajustado por

Funções de Densidade de Probabilidade (PDF).

Caso 2: $U_{s L}=0,61 \mathrm{~m} / \mathrm{s}$ e $U_{s G}=2,55 \mathrm{~m} / \mathrm{s}$.

Figura 5.11 - Evolução do Comprimento da Golfada Ajustado por Funções de Densidade de Probabilidade (PDF).

Caso 3: $U_{s L}=0,61 \mathrm{~m} / \mathrm{s}$ e $U_{s G}=4,64 \mathrm{~m} / \mathrm{s}$.

Figura 5.12 - Evolução da Velocidade Média da Frente e Cauda das Golfadas ao Longo da Tubulação.

Figura 5.13 - Variação do hold-up do Líquido com o Tempo para

Duas Posições. Caso 2: $U_{s L}=0,61 \mathrm{~m} / \mathrm{s}, U_{s G}=2,55 \mathrm{~m} / \mathrm{s}$

Figura 5.14 - Distribuição da Freqüência com o Tempo para duas

Posições. Caso 2: $U_{s L}=0,61 \mathrm{~m} / \mathrm{s}, U_{s G}=2,55 \mathrm{~m} / \mathrm{s}$.

Figura 5.15 - .Distribuição da Freqüência Média no Domínio para os casos 1, 2 e 3, em função da Velocidade de Mistura e da Velocidade Superficial do Líquido

Figura 5.16 - Perfil Instantâneo de Pressão ao Longo do Duto.

Caso 2: $U_{S L}=0,61 \mathrm{~m} / \mathrm{s}, U_{s G}=2,55 \mathrm{~m} / \mathrm{s}$

Figura 5.17 - Coeficiente de Pressão ao Longo do Duto

Figura 5.18 - Perfil Instanteneo de Velocidade do Gas e do Líquido ao Longo do Canal.

Figura 5.19 - Mapa de Padrões de Escoamento da Configuração 2.

Figura 5.20 - Evolução Espacial e Temporal do holdup. Configuração

2. (a) Caso 1: $U_{S L}=0,295 \mathrm{~m} / \mathrm{s}, U_{s G}=0,475 \mathrm{~m} / \mathrm{s}$. (b) Caso 
4: $U_{s L}=0,295 \mathrm{~m} / \mathrm{s}, U_{s G}=0,788 \mathrm{~m} / \mathrm{s}$.

Figura 5.21 - Evolução Espacial e Temporal do holdup. Configuração

2. (a) Caso 2: $U_{s L}=0,393 \mathrm{~m} / \mathrm{s}, U_{s G}=0,475 \mathrm{~m} / \mathrm{s}$. (b) Caso

5: $U_{s L}=0,393 \mathrm{~m} / \mathrm{s}, U_{s G}=0,788 \mathrm{~m} / \mathrm{s}$.

Figura 5.22 - Evolução Espacial e Temporal do holdup. Configuração

2. (a) Caso 3: $U_{s L}=0,516 \mathrm{~m} / \mathrm{s}, U_{s G}=0,475 \mathrm{~m} / \mathrm{s}$. (b) Caso

6: $U_{s L}=0,516 \mathrm{~m} / \mathrm{s}, U_{s G}=0,788 \mathrm{~m} / \mathrm{s}$.

Figura 5.23 - Comparação da Velocidade de Translação da Frente e Cauda da Golfada com a Correlação de Bendiksen (1984).

Figura 5.24 - Evolução da Velocidade Média da Frente e Cauda das Golfadas ao Longo da Tubulação. (a) Caso 1: $U_{S L}=$ $0,295 \mathrm{~m} / \mathrm{s}, U_{s G}=0,475 \mathrm{~m} / \mathrm{s}$. (b) Caso 4: $U_{s L}=0,295$ $\mathrm{m} / \mathrm{s}, U_{s G}=0,788 \mathrm{~m} / \mathrm{s}$ (c) Caso 2: $U_{s L}=0,393 \mathrm{~m} / \mathrm{s}, U_{s G}=$ $0,475 \mathrm{~m} / \mathrm{s}$. (d) Caso 5: $U_{s L}=0,393 \mathrm{~m} / \mathrm{s}, U_{s G}=0,788 \mathrm{~m} / \mathrm{s}$ (e) Caso 3: $U_{s L}=0,516 \mathrm{~m} / \mathrm{s}, U_{s G}=0,475 \mathrm{~m} / \mathrm{s}$. (f) Caso 6: $U_{s L}=0,516 \mathrm{~m} / \mathrm{s}, U_{s G}=0,788 \mathrm{~m} / \mathrm{s}$.

Figura 5.25 - Distribuição do Comprimento da Golfada Líquida e da Bolha na Coordenada $x=9$ m. (a) Golfada Líquida (b) Bolha.

Figura 5.26 - Distribuição PDF do Comprimento da Golfada para $U_{s L}$ $=0,295 \mathrm{~m} / \mathrm{s}$. (a) Caso 1: $U_{s L}=0,295 \mathrm{~m} / \mathrm{s}, U_{s G}=0,475$ $\mathrm{m} / \mathrm{s}$. (b) Caso 4: $U_{s L}=0,295 \mathrm{~m} / \mathrm{s}, U_{s G}=0,788 \mathrm{~m} / \mathrm{s}$.

Figura 5.27 - Distribuição PDF do Comprimento da Golfada para $U_{s L}$ $=0,393 \mathrm{~m} / \mathrm{s}$. (a) Caso 2: $U_{s L}=0,393 \mathrm{~m} / \mathrm{s}, U_{s G}=0,475$ $\mathrm{m} / \mathrm{s}$. (b) Caso 5: $U_{s L}=0,393 \mathrm{~m} / \mathrm{s}, U_{s G}=0,788 \mathrm{~m} / \mathrm{s}$

Figura 5.28 - Distribuição PDF do Comprimento da Golfada para $U_{s L}$ $=0,393 \mathrm{~m} / \mathrm{s}$. (a) Caso 3: $U_{s L}=0,516 \mathrm{~m} / \mathrm{s}, U_{s G}=0,475$ $\mathrm{m} / \mathrm{s}$. (b) Caso 6: $U_{s L}=0,516 \mathrm{~m} / \mathrm{s}, U_{s G}=0,788 \mathrm{~m} / \mathrm{s}$.

Figura 5.29 - Distribuição do Comprimento das Golfadas Líquidas ao Longo da Tubulação. $U_{s G}=0,475 \mathrm{~m} / \mathrm{s}$. (a) Caso 1: $U_{s L}=$ 0,295 m/s (b) Caso 2: $U_{s L}=0,393 \mathrm{~m} / \mathrm{s}$ (c) Caso 3: $U_{s L}=$ $0,516 \mathrm{~m} / \mathrm{s}$. 
Figura 5.30 - Distribuição do Comprimento das Golfadas Líquidas ao Longo da Tubulação. $U_{s G}=0,788 \mathrm{~m} / \mathrm{s}$. (a) Caso 4: $U_{s L}=$ 0,295 m/s (b) Caso 5: $U_{s L}=0,393 \mathrm{~m} / \mathrm{s}$ (c) Caso 6: $U_{s L}=$ $0,516 \mathrm{~m} / \mathrm{s}$.

Figura 5.31 - Variação do Hold-up do Líquido com o Tempo em $\mathrm{x}=9$

m. ( a) Caso 1: $U_{s L}=0,41 \mathrm{~m} / \mathrm{s}$ e $U_{s G}=2,36 \mathrm{~m} / \mathrm{s}$ (b) Caso

3: $U_{s L}=0,61 \mathrm{~m} / \mathrm{s}$ e $U_{s G}=4,64 \mathrm{~m} / \mathrm{s}$ (c) Caso 5: $U_{s L}=$ $0,393 \mathrm{~m} / \mathrm{s}, U_{s G}=0,788 \mathrm{~m} / \mathrm{s}$.

Figura 5.32 - Distribuição da Freqüência com o Tempo Obtido em $x=$ $9 \mathrm{~m}$. (a) Caso 1: $U_{s L}=0,41 \mathrm{~m} / \mathrm{s}$ e $U_{s G}=2,36 \mathrm{~m} / \mathrm{s}$ (b) Caso 3: $U_{s L}=0,61 \mathrm{~m} / \mathrm{s}$ e $U_{s G}=4,64 \mathrm{~m} / \mathrm{s}$ (c) Caso 5: $U_{s L}$ $=0,393 \mathrm{~m} / \mathrm{s}, U_{s G}=0,788 \mathrm{~m} / \mathrm{s}$.

Figura 5.33 - Distribuição da Freqüência Média no Dominio para os Casos da Configuração 2, em Função da Velocidade de Mistura e da Velocidade Superficial do Líquido. 103

Figura 5.34 - Coeficiente de Pressão ao Longo do Duto. Casos 1,2 e 3 


\section{Lista de Símbolos}

A Área da seção transversal da tubulação

A Matriz Jacobiana do sistema característico

B Matriz Jacobiana do sistema característico

D Diâmetro da tubulação

D Vetor coluna do sistema característico

C Matriz Jacobiana do sistema característico

C Número de Courant

$C_{o} \quad$ Parâmetro de distribuição

$f \quad$ Fator de fricção

F Parâmetro adimensional

$\tilde{F} \quad$ Pseudo fluxo convectivo

Fr Número de Froude

$g \quad$ Aceleração da gravidade

$h_{L} \quad$ Altura da superfície do líquido

$K \quad$ Parâmetro adimensional

$L \quad$ Comprimento da unidade básica de golfada

$L_{f} \quad$ Comprimento do filme líquido

$L_{s} \quad$ Comprimento das golfadas

n Número de elementos da mostra

m Número de parâmetros de probabilidade

$P \quad$ Pressão interfacial e da fase gasosa

$R \quad$ Constate do gás

$P_{i} \quad$ Valores do algoritmo TDMA

$Q_{i} \quad$ Valores do algoritmo TDMA

Re Número de Reynolds

$S \quad$ Perímetro molhado

$s \quad$ Coeficiente de correção

$t \quad$ Tempo 
$T$ Temperatura de referência / Parâmetro adimensional

U Velocidade

$U_{d} \quad$ Velocidade de "driff"

$U_{K} \quad$ Velocidade da fase $K$

$U_{M} \quad$ Velocidade da mistura

$U_{s K} \quad$ Velocidade superficial da fase $K$

y Variável aleatória Log-Normal

$x \quad$ Coordenada axial

$X \quad$ Espaço de amostragem

$X^{2} \quad$ Parâmetro de Lockhart Martinelli

\section{Símbolos gregos}

$\alpha \quad$ Fração volumétrica da fase

$\beta$ Ângulo de inclinação da tubulação com respeito à horizontal

$\Delta \quad$ Variação de uma grandeza

$\phi \quad$ Grandeza a ser calculada

$\Phi \quad$ Vetor solução do sistema de autovalores

$\gamma \quad$ Fator de subrelaxação

$\varphi \quad$ Variável aleatória normal

$\varphi_{K} \quad$ Valor médio de uma função relativa à fase $K$

$\varepsilon \quad$ Erro - Padrão

$\mu \quad$ Viscosidade dinâmica / Media das variáveis aleatórias

$v \quad$ Viscosidade cinemática

$v_{s} \quad$ Freqüência das golfadas

$\pi \quad$ Constante $\mathrm{Pi}$

$\xi \quad$ Parâmetro geométrico auxiliar

$\rho \quad$ Massa específica

$\sigma \quad$ Tensão superficial / Desvio padrão das variáveis aleatórias

$\tau \quad$ Tensão de cisalhamento

$\forall \quad$ Volume 


\section{Subscritos}

$b \quad$ Translação da cauda da golfada

$e, w \quad$ Faces leste e oeste do volume de controle principal

E Referente ao centro do volume principal de controle a leste entrada Entrada da tubulação

$G \quad$ Fase gasosa

I lésimo ponto nodal

i Interface

$L \quad$ Fase líquida

M Mistura

max Máximo valor

$N \quad$ Número total de nós no domínio / medidas realizadas

$n$ n-ésima medida

$P \quad$ Referente ao centro do volume de controle principal

$r \quad$ Relativa

ref Referência

$s \quad$ Referente a "slug", ou golfada

saída Saída da tubulação

$t$ Translação da frente da golfada

w Parede da tubulação

$W \quad$ Referente ao centro do volume principal de controle a leste

\section{Sobrescritos}

$o$ Referente ao passo de tempo anterior

* Referente à iteração anterior

flag Indicador de formação de slug

ref Referência

1 Referente a uma grandeza aproximada mediante esquema Upwind

Referente a uma grandeza aproximada

* $\quad$ Referente à iteração anterior 